\title{
A power sharing algorithm for a hybrid energy storage system based on batteries
}

\author{
Francisco Díaz-González, Mònica Aragüés-Peñalba, Francesc Girbau-Llistuella, Marc Llonch-Masachs, \\ Andreas Sumper
}

\begin{abstract}
This paper presents a method to operate a hybrid Energy Storage System (ESS) based on a heterogenous grouping of battery types. In particular, the system is composed by a lithium-ion battery pack and a lead-acid one. The operational method is based on an optimization Non Linear Problem (NLP), which takes the name of power sharing algorithm. The aim of this algorithm is to fit the output of the hybrid ESS to the profile setpoint while minimizing degradation and taking advantage of main performance of each battery type. An exemplary study case is offered so as to proof the validity of the algorithm.
\end{abstract}

Index Terms-Hybrid Energy Storage System, battery, power electronics, optimization algorithm.

\section{INTRODUCTION}

During the last decades, an increasing number of Energy Storage Systems (ESSs) are being deployed in the electrical power system premises, including customer, distribution and transmission installations [1]. From a general perspective, they are facilitating the integration of large amounts of renewable energy sources, helping to operate systems with higher variability and uncertainty both in the generation and demand side; contributing to ensure their equilibrium.

In particular, the services that energy storage technologies can offer in renewable-based power system have been addressed in [2], classifying them according to the power and energy ratings of the ESS and the type of beneficiary. At distribution level, and in benefit of DSOs, storage can facilitate power quality improvement, congestion alleviation and security of supply. For customers, ESS enable self-consumption, off-grid operation and active demand management. At transmission level, ESS also offer grid ancillary services (primary and secondary power reserves, voltage control, power ramprate limitation and black start capability) and enhance the controllability of power generation. Therefore, based on all the grid services ESS can offer, they can play a key role in the transition towards a decarbonized energy system [3]. However, some planning and operation challenges to integrate them properly remain and have to cope with the existing regulatory barriers.

Francisco Díaz-González, Mònica Aragüés-Peñalba, Francesc GirbauLlistuella, Marc Llonch-Masachs and Andreas Sumper are with Centre d'Innovació Tecnològica en Convertidors Estàtics i Accionaments (CITCEA-UPC), Departament d'Enginyeria Elèctrica, Universitat Politècnica de Catalunya EU d'Enginyeria Tècnica Industrial de Barcelona, Avda Diagonal 647, Pl. 2, 08028 Barcelona, Spain (e-mail: francisco.diazgonzalez@upc.edu).
The present study focuses on the integration of an ESS in low voltage distribution grids for enhancing the efficiency and the hosting capacity of distribution networks, and this is framed in the research project H2020 RESOLVD [4]. For this purpose, a hybrid ESS is being proposed. Hybridisation is because the ESS combines a heterogeneous grouping of battery types in a single unit. In particular, in this study the hybrid ESS is composed by two different battery types: one based on lithium-ion batteries and the other based on lead-acid ones. The motivation of combining those technologies is due to the fact that the associated investment can be diminished (as a difference with lithium-ion, lead-acid batteries are cheap, so part of storage requirements for the ESS can be fulfilled at low cost). However, the performance of lead-acid batteries is not as good as for lithium-ion in terms of cyclability and efficiency. Thus, optimal operational capability is needed to operate simultaneously and in a complementary way both battery technologies, so the performance of the hybrid ESS as a whole, is not diminished. Such optimal operation is enabled by a novel power sharing algorithm and this is the main contribution of the paper. This algorithm distributes the power demand received by the ESS from the network operation among the battery types embedded in, considering different aspects including the performance and degradation of each type. The description of the power electronics-based power conversion system integrating the batteries into the ESS and interfacing them with the external network is out of the scope in this paper.

\section{POWER SHARING OPTIMIZATION FORMULATION}

The optimization algorithm is a Non-Linear-Problem (NLP). Input data is introduced in subsection II-A, decision variables in II-B, and constraints and function objective in II-C.

\section{A. Input data}

The input data for the mathematical problem is divided into sets and parameters, see Table I.

\section{B. Decision variables}

The NLP solves with the optimal way to charge and discharge batteries. Such vision is translated into the decision variables in Table II. 
TABLE I

INPUT DATA

\begin{tabular}{|c|c|}
\hline Item & Description \\
\hline$T$ & Set for time period $T=t_{0}, . ., t_{f}$. \\
\hline$I$ & Set for battery units $I=i_{0}, \ldots, i_{f}$. \\
\hline$P_{r_{i}}$ & Rated power for battery $i$, in $\mathrm{kW}$. \\
\hline$E_{r_{i}}^{2}$ & Rated energy for battery $i$, in $\mathrm{kWh}$. \\
\hline$P_{\text {tott }}$ & Power demand for the ESS at time $t$, in $\mathrm{kW}$ \\
\hline$U_{t}$ & $\begin{array}{l}\text { Boolean parameter indicating the sign of } P_{t o t_{t}} \text {, ( } 1 \text { for } \\
\text { positive; } 0 \text { otherwise). }\end{array}$ \\
\hline$\varepsilon$ & $\begin{array}{l}\text { Maximum discrepancy between initial and final state of } \\
\text { charge for the set of batteries, in } \mathrm{kWh}\end{array}$ \\
\hline$\delta$ & $\begin{array}{l}\text { Maximum discrepancy between } P_{t t_{t}} \text { and the actual } \\
\text { output / input for the ESS, in p.u. }\end{array}$ \\
\hline$S O C_{\max _{i}}$ & Maximum admissible state of charge for battery $i$, in p.u. \\
\hline$S O C_{\text {min }_{i}}$ & Minimum admissible state of charge for battery $i$, in p.u. \\
\hline$\eta_{i n_{i}}$ & Charging efficiency for battery $i$, in p.u. \\
\hline$\eta_{\text {out }_{i}}$ & Discharging efficiency for battery $i$, in p.u. \\
\hline$C_{p_{i}}$ & $\begin{array}{l}\text { Degradation cost in terms of the power magnitude ex- } \\
\text { changed by battery } i \text {, in p.u. }\end{array}$ \\
\hline$C_{d_{i}}$ & $\begin{array}{l}\text { Degradation cost in terms of depth of discharge experi- } \\
\text { enced by battery } i \text {, in p.u. }\end{array}$ \\
\hline$\alpha$ & $\begin{array}{l}\text { Weight for optimization objective of minimizing the } \\
\text { discrepancy between power demand for the ESS and } \\
\text { actual output, in p.u. }\end{array}$ \\
\hline$\beta$ & $\begin{array}{l}\text { Weight for optimization objective of minimizing degra- } \\
\text { dation because of excessive power, in p.u. }\end{array}$ \\
\hline$\gamma$ & $\begin{array}{l}\text { Weight for optimization objective of minimizing degra- } \\
\text { dation because of excessive power ramp, in p.u. }\end{array}$ \\
\hline
\end{tabular}

TABLE II

DECISION VARIABLES

\begin{tabular}{ll}
\hline Item & Description \\
\hline$p_{i, t}$ & Power exchanged by battery $i$ at time $t$, in $\mathrm{kW}$. \\
$e_{i n_{i, t}}$ & Energy consumed by battery $i$ at time $t$, in $\mathrm{kWh}$. \\
$e_{o u t_{i, t}}$ & Energy injected by battery $i$ at time $t$, in $\mathrm{kWh}$. \\
$d p_{i, t}$ & Ramp for the power exchanged by battery $i$ at time $t$, in \\
& $\mathrm{kW} / \mathrm{h}$. \\
soc $_{i, t}$ & State of charge of battery $i$ at time $t$, in $\mathrm{kWh}$. \\
\hline
\end{tabular}

\section{Problem constraints and function objective}

The state of charge for the battery $i$ at time $t$ is expressed in terms of the energy absorbed and injected $-e_{i n_{i, t}}$ and $e_{o u t_{i, t}}$ respectively-, and the corresponding charging and discharging efficiencies, $\eta_{i n_{i}}$ and $\eta_{\text {out }}$. Thus,

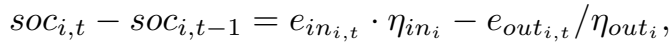

and $s o c_{i, t}$ should be maintained within predetermined limits, so

$$
\begin{array}{ll}
\operatorname{soc}_{i, t} \leq S O C_{\max _{i}} \cdot E_{r_{i}} & \forall i \in I, t \in T, \\
\operatorname{soc}_{i, t} \geq S O C_{\text {min }_{i}} \cdot E_{r_{i}} & \forall i \in I, t \in T .
\end{array}
$$

From the energy charged and discharged for the battery $i$ at time $t$, the associated average power $p_{i, t}$ developed in this time step can be derived as

$$
p_{i, t} \cdot T_{s}=e_{i n_{i, t}}-e_{\text {out }}{ }_{i, t} \quad \forall i \in I, t \in T .
$$

Power $p_{i, t}$ should not exceed the ratings for each battery $i$. This is represented as

$$
e_{\text {in }_{i, t}}+e_{\text {out }_{i, t}} \leq p_{r_{i}} \cdot T_{s} \quad \forall i \in I, t \in T \text {. }
$$

Then, constraint (6) ensures that the sign for the power developed by battery $i$ at time $t$ is coherent with that for the power setpoint $P_{t_{\text {tot }}}$,

$$
P_{\text {tot }_{t}} \cdot \sum_{i} p_{i, t} \geq 0 \quad \forall t \in T .
$$

In addition, the power exchanged by the set of batteries at time $t$ should be similar to the setpoint $P_{\text {tot }_{t}}$. A margin is provided though, because the ESS should have some flexibility to adjust their output so it can compensate power losses. Such flexibility is represented by an admissible error to $P_{t o t_{t}}$, expressed by parameter $\delta$,

$$
\begin{array}{cc}
\delta \cdot U_{t} \cdot P_{\text {tot }_{t}} \geq U_{t} \cdot\left(P_{\text {tot }_{t}}-\sum_{i} p_{i, t}\right) & \forall t \in T, \\
-\delta \cdot U_{t} \cdot P_{\text {tot }_{t}} \leq U_{t} \cdot\left(P_{\text {tot }_{t}}-\sum_{i} p_{i, t}\right) & \forall t \in T, \\
\delta \cdot\left(1-U_{t}\right) \cdot P_{\text {tot }_{t}} \leq\left(1-U_{t}\right) \cdot\left(P_{\text {tot }_{t}}-\sum_{i} p_{i, t}\right) & \forall t \in T, \\
-\delta \cdot\left(1-U_{t}\right) \cdot P_{\text {tot }_{t}} \geq\left(1-U_{t}\right) \cdot\left(P_{\text {tot }_{t}}-\sum_{i} p_{i, t}\right) \quad \forall t \in T .
\end{array}
$$

Constraint (11) ensures that the difference between the initial and final state of charge for the set of batteries (in $\mathrm{kWh}$ ) is almost equal -assuming a small discrepancy represented by parameter $\varepsilon$, in $\mathrm{kWh}$ - to the energy balance derived from the power setpoint profile for the whole time period of analysis,

$$
\left(\sum_{i}{s o c_{i, t_{f}}}-\sum_{i} s o c_{i, t_{0}}-\sum_{t} P_{t t_{t}} \cdot T_{s}\right)^{2} \leq \varepsilon .
$$

Finally, constraints (12) to (14) ensures the non-negativity of variables,

$$
\begin{array}{ll}
\operatorname{soc}_{i, t} \geq 0 & \forall i \in I, t \in T, \\
e_{\text {in }_{i, t}} \geq 0 & \forall i \in I, t \in T, \\
e_{\text {out }_{i, t}} \geq 0 & \forall i \in I, t \in T .
\end{array}
$$

The optimization criteria are two-fold. Firstly, the discrepancy between the power demand for the ESS and the actual power exchanged should be minimized, regardless the power losses. Such discrepancy is quantified by term $z_{p}$, which is formulated as,

$$
z_{p}=\sum_{t}\left(\frac{P_{\text {tot }_{t}}-\sum_{i} p_{i, t}}{\sum_{i} P_{r_{i}}}\right)^{2} .
$$

As can be noted, the square of the difference between $P_{\text {tot }_{t}}$ and $\sum_{i} p_{i, t}$ is computed in equation (15). This is a strategy to fit the response of the ESS to the power setpoint at all times. The second optimization criterion is to operate each of the batteries so the associated degradation can be minimized. This is related to two circumstances: $i$ ) the power exchanged; and ii) the depth of discharge. The higher the magnitude of such quantifiers, the higher the degradation for batteries. In general terms, thus, it is advisable to operate batteries as derated as possible and performs smoothed power profiles also. The advantage of hybridizing the ESS is to use the lithium-ion battery when answering to high and prolonged power demand; 
and the lead-acid battery for less stringent power profiles. This way, the degradation for lead-acid battery can be minimized, and the ESS can still provide good dynamic response through the resiliency of lithium-ion battery against degradation.

To penalise the degradation of batteries in terms of power rating, term $z_{d 1}$ is included and formulated as

$$
z_{d 1}=\sum_{i, t}\left(\frac{p_{i, t}}{P_{r_{i}}} \cdot C_{p_{i}}\right)^{2} .
$$

To penalise the degradation of batteries in terms of depth of discharge, term $z_{d 2}$ is included in the objective function and formulated as,

$$
z_{d 2}=\sum_{i, t}\left(\frac{s o c_{i, t}}{E_{r_{i}}} \cdot C_{d_{i}}\right)^{2} .
$$

At the end, the problem turns into a multi-objective criteria which objective function is

$$
z=\alpha \cdot \frac{z_{p}}{Z_{p}^{*}}+\beta \cdot \frac{z_{d 1}}{Z_{d 1}^{*}}+\gamma \cdot \frac{z_{d 2}}{Z_{d 2}^{*}},
$$

where $\alpha, \beta$ and $\gamma$ are weighting factors for each of the optimization criteria, and the sum of all three factors is one. In addition, each of the optimization criterion $z_{p}, z_{d 1}$ and $z_{d 2}$ are divided by the value it takes $z$ when considering each one as the unique criterion for optimization $\left(Z_{p}^{*}, Z_{d 1}^{*}\right.$ and $Z_{d 2}^{*}$, respectively). Thus, each of the optimization criterion can be summed and compared between them, since levelized.

\section{STUDY CASE}

This section presents the study case so as to proof the performance of the power sharing algorithm. Input data is introduced in subsection III-A and optimization results are summarized in III-B.

\section{A. Input data}

The hybrid storage system is composed by two battery types: a lead-acid pack and a lithium-ion pack. Both battery packs have the same ratings in terms of energy and power. These two technologies -lithium-ion and lead-acid types- are very different in terms of technical characteristics and costs, so they are good candidates for configuring a hybrid system because of their complementarity. Data characterizing battery packs is presented in Table III.

\section{TABLE III}

CHARACTERISTICS OF BATTERY PACKS

\begin{tabular}{lll}
\hline Item & Lithium-ion & Lead-acid \\
\hline$P_{r_{i}}$ & $5 \mathrm{~kW}$ & $5 \mathrm{~kW}$ \\
$E_{r_{i}}$ & $12 \mathrm{kWh}$ & $12 \mathrm{kWh}$ \\
$S O C_{\max _{i}}$ & 0.99 p.u. & 0.99 p.u. \\
$S O C_{\min _{i}}$ & 0.1 p.u. & 0.3 p.u. \\
$\eta_{i n_{i}}$ & 0.85 p.u. & 0.72 p.u. \\
$\eta_{o u t}$ & 0.85 p.u. & 0.72 p.u. \\
$C_{p_{i}}$ & 0.125 p.u. & 1.0 p.u. \\
$C_{d_{i}}$ & 0.2 p.u. & 1.0 p.u. \\
\hline
\end{tabular}

As shown, the total energy capacity for the ESS is $24 \mathrm{kWh}$. The maximum state of charge for both batteries almost reaches
$100 \%$ but the minimum depends on their type, being the lithium-ion pack that widthstands much deeper discharges than the lead-acid pack. The charging and discharging efficiency is also better for lithium-ion pack.

The last two terms in the table characterizing batteries refer to their resiliency against degradation, terms $C_{p_{i}}$ and $C_{d_{i}}$. Degradation in terms of power charge and discharge rates is considered as several times higher for lead-acid with respect to lithium-ion. A proof is that manufacturers, in their datasheets, recommend discharge rates are usually around $0.1 \mathrm{C}$ or $0.2 \mathrm{C}$ (so at $10 \%$ or $20 \%$ of the nominal design current for the battery) so as to maximize useful life; while this rate can be $0.7 \mathrm{C}$ or even $1 \mathrm{C}$ for lithium-ion batteries, so around 8 times more on average [5], [6], [7]. In turn, degradation in terms of depth of discharge is also much higher for the lead-acid pack than for the lithium-ion one. In particular, it is weighted as 5 times higher according to Table III, parameter $C_{d_{i}}$.

For the purposes of the study case, the hybrid ESS should follow an arbitrary power output profile, named in Table I as $P_{\text {tot }_{t}}$. Such power profile is configured by 57601 -minute time steps, so $T s=0.0167 \mathrm{~h}$. Thus, it represents the power demand profile for the battery for 4 days. The maximum charge variation for an ideal ESS (100\% efficiency, no losses) to fulfill such power demand would be $16 \mathrm{kWh}$. This way, the proposed sizing for the ESS ( $24 \mathrm{kWh})$ should be enough to answer to $P_{\text {tot }_{t}}$. However, using just 1 battery type would not be enough, since rated at $12 \mathrm{kWh}$. Thus, the selected scenario, forces the simultaneous operation of the two battery packs. The power sharing algorithm, will solve the optimal way to do that according to the technical characteristics and limitations of each battery type.

Because of batteries are not ideal (they present losses in charging and discharging) it may be not possible to strictly follow $P_{t_{t} t_{t}}$. Some flexibility should be provided so that losses can be compensated in time. Such flexibility is weighted by parameter $\delta$. In the present case, this is fixed to 0.35 , indicating an admissible discrepancy of the output of the ESS up to $\pm 35 \%$ with respect to $P_{t t_{t}}$. Respecting such maximum thereshold, the power sharing algorithm will minimize the discrepancy considering the needs for each battery type to compensate losses.

\section{B. Optimization results}

This section presents some exemplary results with the aim of showing the performance of the power sharing algorithm. In the following results, the criterion of minimizing the error between the output of the ESS and its power profile setpoint has the same importance (i.e. the same weight) as the criterion of minimizing the degradation of the batteries. This implies that weighting parameter $\alpha$ is set to 1 ; and the sum of weighting factors for degradation (parameters $\beta$ and $\gamma$ ) are also 1 . In this particular case, $\beta$ and $\gamma$ are both set to 0.5 .

Figure 1 firstly presents the performance of the ESS while fitting its output to the power profile setpoint. Positive values means to absorb power from the grid. As can be noted, while absorbing power, the power profile developed by the ESS $\left(\sum_{i, t} p_{i, t}\right)$ mostly fits with the setpoint $\left(P_{t o t_{t}}\right)$. However, while 


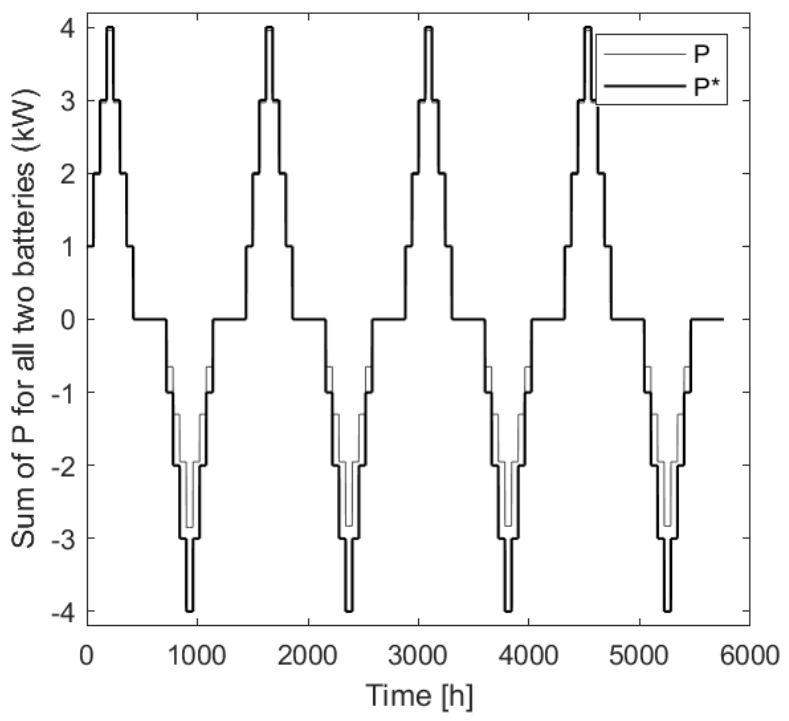

Fig. 1. Power profile setpoint and the actual power exchanged by the ESS.

injecting power to the grid, the output of the ESS is lower than requested. This difference though, is within admissible limits (35\%, as set by parameter $\delta$ ). Such difference is because the batteries need some flexibility to compensate losses: it is not possible to strictly comply with power setpoint at all times.

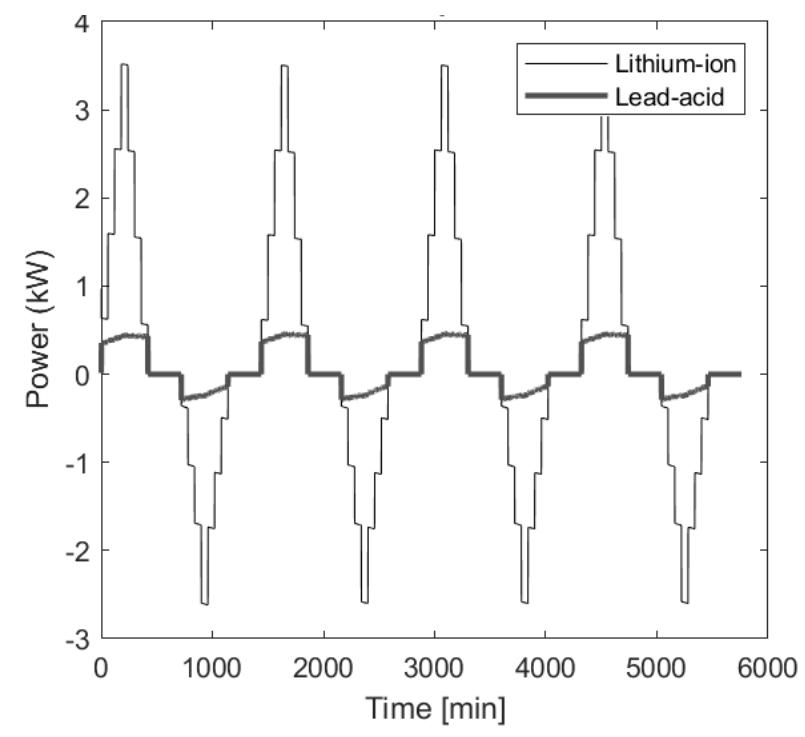

Fig. 2. Power input / output for each of the battery types of the ESS.

Figure 2 then depicts how the lithium-ion and the leadacid battery contribute to the achievement of the power profile developed by the ESS as a whole. As can be noted, the lithiumion battery, since being the one with best performance in terms of efficiency and degradation, is holding most of power demand, both in terms of power magnitude and power ramp. The lead-acid battery, on the other hand, is developing much lower power for reduced degradation.

Finally, Figure 3 presents the state of charge variation for both batteries while answering to the power profile setpoint. As can be noted, most of the energy storage capacity is utilized for

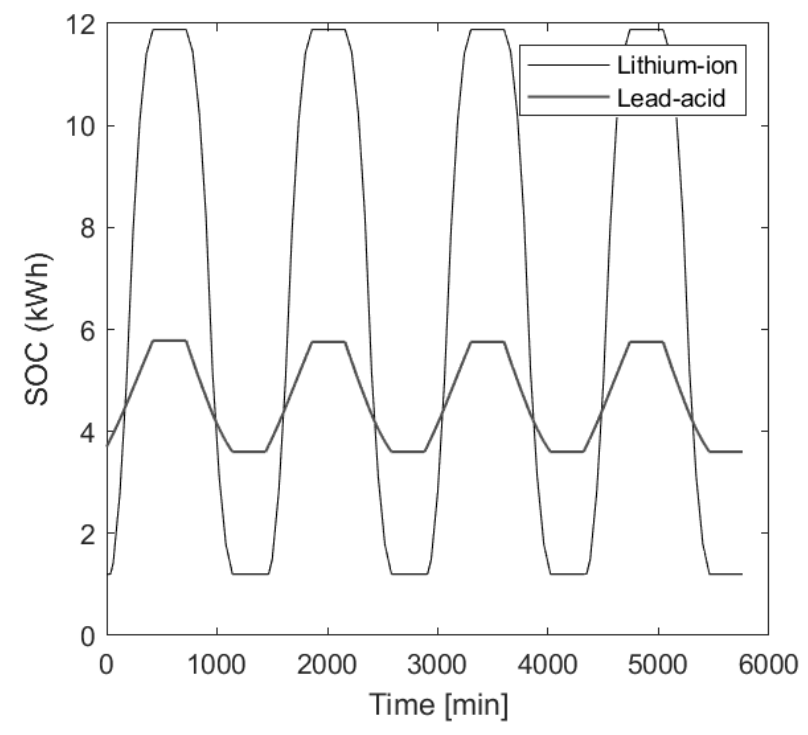

Fig. 3. State of charge of each of the battery types of the ESS.

the lithium-ion battery. In addition, the state of charge variation presents high ramping, in coherence with the significant power developed in time. Conversely, the lead-acid battery is operated smoothly and within a limited state of charge range.

\section{CONCLUSIONS AND FURTHER WORK}

This paper presented a method to operate synergistically a set of batteries of different type. This method was based on an optimization NLP. The advantage of the proposed hybrid ESS along with the mentioned operational optimization, among state of the art ESSs based on solely one battery type is that it is possible to still obtain excellent service performance while minimizing investment costs. The operational optimization, called power sharing algorithm, enables the operation of each of the batteries for minimum degradation, thus maximizing their lifespan. The exemplary proposed study case, validated the formulation of the power sharing algorithm.

Next research goals are around the implementation and further testing of the algorithm in an actual hybrid ESS to be validated in field.

\section{ACKNOWLEDGMENTS}

This work has been supported by the European Union's Horizon 2020 programme under the grant agreement number 773715 .

\section{REFERENCES}

[1] Energy Storage Association "Advanced energy storage in integrated resource planning. 2018 update". [Online]. Available: http://energystorage. org/IRP [Accessed: Jun. 19, 2019]

[2] F. Díaz-González, E. Bullich-Massagué, C. Vitale, M. Gil-Sáanchez, M. Araguiés-Peñalba, F. Girbau-Llistuella, "Services of energy storage technologies in renewable-based power systems," presented at IberoAmerican Congress of Smart Cities, Soria, Spain, 2018

[3] F. Díaz-González, O. Gomis-Bellmunt and A. Sumper, Energy storage in power systems. John Wiley and Sons, 2016

[4] H2020 RESOLVD project, Renewable Pentration Levered by Efficient Low Voltage Distribution Grids. [Online]. Available: https://resolvd.eu/. [Accessed: Mar. 1, 2019] 
[5] Power Sonic Corporation website, "PDC-121000 battery datasheet". [Online]. Available: https://www.power-sonic.com/product/pdc-121000/. [Accessed: Mar. 1, 2019]

[6] Panasonic website, "Lithium-ion batteries datasheet". [Online]. Available: https://na.industrial.panasonic.com/products/batteries/ rechargeable-batteries/lithium-ion. [Accessed: Mar. 1, 2019]

[7] Kokam website, "Lithium-ion batteries". [Online]. Available: http:// kokam.com/cell/. [Accessed: Mar. 1, 2019]

[8] M. Dubarry, A. Devie, "Battery durability and reliability under electric utility grid operations: Representative usage aging and calendar aging". Journal of Energy Storage, vol. 18, pp. 185-195, 2018

[9] M. Dubarry, A. Devie, K. Stein, M. Tun, M. Matsuura, R. Rocheleau, "Battery Energy Storage System battery durability and reliability under electric utility grid operations: Analysis of 3 years of real usage". Journal of Power Sources, vol. 338, pp. 65-73, 2018

[10] L. Barelli, G. Bidini, F. Bonucci, L. Castellini, A. Fratini, F. Gallorini, A. Zuccari, "Flywheel hybridization to improve battery life in energy storage systems coupled to RES plants". Energy, article in press. DOI: 10.1016/j.energy.2019.02.143 